\title{
Wilks' Factorization of the Complex Matrix Variate Dirichlet Distributions
}

\author{
Xinping CUI, Arjun K. GUPTA, \\ and Daya K. NAGAR \\ Department of Statistics \\ University of California-Riverside \\ Riverside, CA 92521, USA \\ xinping. cui@ucr.edu \\ Department of Mathematics and Statistics \\ Bowling Green State University \\ Bowling Green, Ohio 43403-0221, USA \\ gupta@bgnet.bgsu. edu \\ Departamento de Matemáticas \\ Universidad de Antioquia \\ Medellín, A. A. 1226, Colombia \\ nagar@matematicas.udea.edu.co
}

Recibido: 27 de julio de 2004

Aceptado: 17 de diciembre de 2004

\begin{abstract}
In this paper, it has been shown that the complex matrix variate Dirichlet type I density factors into the complex matrix variate beta type I densities. Similar result has also been derived for the complex matrix variate Dirichlet type II density. Also, by using certain matrix transformations, the complex matrix variate Dirichlet distributions have been generated from the complex matrix beta distributions. Further, several results on the product of complex Wishart and complex beta matrices with a set of complex Dirichlet type I matrices have been derived.
\end{abstract}

Key words: beta distribution, complex random matrix, Dirichlet distribution, Jacobian, complex multivariate gamma function, transformation, Wishart distribution.

2000 Mathematics Subject Classification: Primary 62H10; Secondary 62E20

The research work of DKN was supported by the Comité para el Desarrollo de la Investigación, Universidad de Antioquia research grant no. IN387CE. 


\section{Introduction}

The random variables $x_{1}, \ldots, x_{n}$ are said to have the univariate Dirichlet type I distribution with parameters $a_{1}, \ldots, a_{n} ; a_{n+1}$ if their joint probability density function (p.d.f.) is given by

$$
\begin{aligned}
& \frac{\Gamma\left(\sum_{i=1}^{n+1} a_{i}\right)}{\prod_{i=1}^{n+1} \Gamma\left(a_{i}\right)} \prod_{i=1}^{n} x_{i}^{a_{i}-1}\left(1-\sum_{i=1}^{n} x_{i}\right)^{a_{n+1}-1}, \\
& \quad 0<x_{i}<1, \quad i=1, \ldots, n, \quad \sum_{i=1}^{n} x_{i}<1,
\end{aligned}
$$

where $a_{i}>0, i=1, \ldots, n+1$. The random variables $y_{1}, \ldots, y_{n}$ are said to have the univariate Dirichlet type II distribution with parameters $b_{1}, \ldots, b_{n} ; b_{n+1}$ if their joint p.d.f. is given by

$$
\frac{\Gamma\left(\sum_{i=1}^{n+1} b_{i}\right)}{\prod_{i=1}^{n+1} \Gamma\left(b_{i}\right)} \prod_{i=1}^{n} y_{i}^{b_{i}-1}\left(1+\sum_{i=1}^{n} y_{i}\right)^{-\sum_{i=1}^{n+1} b_{i}}, \quad 0<y_{i}<\infty, \quad i=1, \ldots, n
$$

where $b_{i}>0, i=1, \ldots, n+1$. The Dirichlet type II density (2) can be obtained from (1) by using the transformation $y_{i}=\left(1-\sum_{i=1}^{n} x_{i}\right)^{-1} x_{i}, i=1, \ldots, n$. For this reason (2) is also known as the inverted Dirichlet density.

Wilks [24] showed that the variates $u_{1}=x_{1}, u_{2}=x_{2}\left(1-x_{1}\right), \ldots, u_{n}=$ $x_{n}\left(1-x_{1}-\cdots-x_{n-1}\right)$ are independently distributed. Tan [22], using certain results on marginal and conditional distributions, derived similar results for the matrix variate Dirichlet type I matrices. He showed that the matrix variate Dirichlet type I density factors in into the matrix variate beta type I densities. Recently, Gupta and Nagar [9], using matrix transformation, derived Wilks' factorization for matrix Dirichlet type I and matrix Dirichlet type II distributions.

The matrix variate Dirichlet distributions have been studied by several authors (see, for example, Olkin and Rubin [18], Tan [22], Javier and Gupta [12], and Gupta and Nagar [8]). An extensive review on the matrix variate Dirichlet distribution is available in Gupta and Nagar [9].

In this article we derive Wilks' factorization of the complex matrix variate Dirichlet distributions. We will also show that using certain matrix transformations one can generate the complex matrix variate Dirichlet distributions from the complex matrix variate beta distributions.

The complex matrix variate Dirichlet distributions were defined and studied by Tan $[21]$.

The complex matrix variate distributions play an important role in various fields of research. Applications of complex random matrices can be found in multiple time series analysis, nuclear physics and radio communications (Carmeli [3], Krishnaiah [15], 
Mehta [16], and Smith and Gao [19]). Bronk [1] has shown that under certain conditions the distribution of the energy levels of atomic nuclei is the distribution of the roots of a complex random matrix. Brillinger [2] has shown that the asymptotic distribution of the matrix of spectral densities of a strictly stationary time series is complex Wishart. A number of results on the distribution of the complex random matrices have also been derived. The complex matrix variate Gaussian distribution was introduced by Wooding [25], Turin [23], and Goodman [6]. The complex Wishart distribution was studied by Goodman [6,7], Srivastava [20], Hayakawa [10], and Chikuse [4]. James [11] and Khatri [13] derived the complex central as well as the noncentral matrix variate beta distributions. Distributional results on quadratic forms involving complex normal variables were given by Khatri [14] and Conradie and Gupta [5]. Nagar and Arias [17] defined the complex matrix Cauchy distribution and studied its properties. They also showed that quadratic forms in partitioned Cauchy matrix follow complex matrix variate Dirichlet type I distribution. Systematic treatment of the distributions of the complex random matrices was given by Tan [21] which included the Gaussian, Wishart, beta, and Dirichlet distributions.

\section{Complex matrix variate Dirichlet distributions}

In this section we will define complex matrix variate Dirichlet type I and II distributions and derive them using complex Wishart matrices. We first state the following notations and results that will be utilized in this and subsequent sections. Let $A=\left(a_{i j}\right)$ be an $m \times m$ matrix of complex numbers. Then, $A^{\prime}$ denotes the transpose of $A, \bar{A}$ denotes the conjugate of $A, A^{H}$ denotes the conjugate transpose of $A$, $\operatorname{tr}(A)=a_{11}+\cdots+a_{m m}, \operatorname{etr}(A)=\exp (\operatorname{tr}(A)), \operatorname{det}(A)$ denotes the determinant of $A$, $A=A^{H}>0$ means that $A$ is Hermitian positive definite, and $A^{\frac{1}{2}}$ denotes the unique Hermitian positive definite square root of $A=A^{H}>0$.

Definition 1.1. The complex multivariate gamma function, denoted by $\tilde{\Gamma}_{m}(\alpha)$, is defined by

$$
\tilde{\Gamma}_{m}(\alpha)=\int_{X=X^{H}>0} \operatorname{etr}(-X) \operatorname{det}(X)^{\alpha-m} d X, \quad \operatorname{Re}(\alpha)>m-1 .
$$

By evaluating the integral in (3), the complex multivariate gamma function can be expressed as product of ordinary gamma functions

$$
\tilde{\Gamma}_{m}(\alpha)=\pi^{m(m-1) / 2} \prod_{i=1}^{m} \Gamma(\alpha-i+1), \quad \operatorname{Re}(\alpha)>m-1 .
$$

Definition 1.2. The complex multivariate beta function, denoted by $\tilde{B}_{m}(a, b)$, is defined by

$$
\tilde{B}_{m}(a, b)=\int_{0<X=X^{H}<I_{m}} \operatorname{det}(X)^{a-m} \operatorname{det}\left(I_{m}-X\right)^{b-m} d X
$$


where $\operatorname{Re}(a)>m-1$ and $\operatorname{Re}(b)>m-1$.

The complex multivariate beta function $\tilde{B}_{m}(a, b)$ can be expressed in terms of complex multivariate gamma functions

$$
\tilde{B}_{m}(a, b)=\frac{\tilde{\Gamma}_{m}(a) \tilde{\Gamma}_{m}(b)}{\tilde{\Gamma}_{m}(a+b)}=\tilde{B}_{m}(b, a)
$$

Substituting $X=\left(I_{m}+Y\right)^{-1} Y$ in (4) with the Jacobian $J(X \rightarrow Y)=J((d X) \rightarrow(d Y))$ $=\operatorname{det}\left(I_{m}+Y\right)^{-2 m}$, we get an equivalent integral representation for the complex multivariate beta function as

$$
\tilde{B}_{m}(a, b)=\int_{Y=Y^{H}>0} \operatorname{det}(Y)^{a-m} \operatorname{det}\left(I_{m}+Y\right)^{-(a+b)} d Y .
$$

Next, we give definitions of complex Wishart, complex beta and complex Dirichlet distributions (Goodman [6], Tan [21]).

Definition 1.3. An $m \times m$ random Hermitian positive definite matrix $X$ is said to follow the complex Wishart distribution, denoted as $X \sim \mathbb{C} W_{m}(n, \Sigma)$, if its p.d.f. is given by

$$
\left\{\tilde{\Gamma}_{m}(n) \operatorname{det}(\Sigma)^{n}\right\}^{-1} \operatorname{etr}\left(-\Sigma^{-1} X\right) \operatorname{det}(X)^{n-m}, \quad \Sigma=\Sigma^{H}>0, \quad n>m-1 .
$$

Definition 1.4. An $m \times m$ random Hermitian positive definite matrix $X$ is said to have the complex matrix variate Beta type I distribution with parameters $(a, b)$, denoted as $X \sim \mathbb{C} B_{m}^{I}(a, b)$, if its p.d.f. is given by

$$
\left\{\tilde{B}_{m}(a, b)\right\}^{-1} \operatorname{det}(X)^{a-m} \operatorname{det}\left(I_{m}-X\right)^{b-m}, \quad 0<X=X^{H}<I_{m},
$$

where $a>m-1$ and $b>m-1$.

Definition 1.5. An $m \times m$ random Hermitian positive definite matrix $Y$ is said to have the complex matrix variate Beta type II distribution with parameters $(a, b)$, denoted as $Y \sim B_{m}^{I I}(a, b)$, if its p.d.f. is given by

$$
\left\{\tilde{B}_{m}(a, b)\right\}^{-1} \operatorname{det}(Y)^{a-m} \operatorname{det}\left(I_{m}+Y\right)^{-(a+b)}, \quad Y=Y^{H}>0,
$$

where $a>m-1$ and $b>m-1$.

Note that if $X \sim \mathbb{C} B_{m}^{I I}(a, b)$, then $(I+X)^{-1} \sim \mathbb{C} B_{m}^{I}(b, a)$ and $\left(I_{m}+X\right)^{-1} X \sim$ $\mathbb{C} B_{m}^{I}(a, b)$.

Definition 1.6. The $m \times m$ random Hermitian positive definite matrices $X_{1}, \ldots, X_{n}$ are said to have the complex matrix variate Dirichlet type I distribution with parameters $\left(a_{1}, \ldots, a_{n} ; a_{n+1}\right)$, denoted by $\left(X_{1}, \ldots, X_{n}\right) \sim \mathbb{C} D_{m}^{I}\left(a_{1}, \ldots, a_{n} ; a_{n+1}\right)$, if their joint p.d.f. is given by

$$
\frac{\tilde{\Gamma}_{m}\left(\sum_{i=1}^{n+1} a_{i}\right)}{\prod_{i=1}^{n+1} \tilde{\Gamma}_{m}\left(a_{i}\right)} \prod_{i=1}^{n} \operatorname{det}\left(X_{i}\right)^{a_{i}-m} \operatorname{det}\left(I_{m}-\sum_{i=1}^{n} X_{i}\right)^{a_{n+1}-m}
$$

where $I_{m}-\sum_{i=1}^{n} X_{i}$ is Hermitian positive definite and $a_{i}>m-1$, for $i=1, \ldots, n+1$. 
Definition 1.7. The $m \times m$ Hermitian positive definite random matrices $Y_{1}, \ldots, Y_{n}$ are said to have the complex matrix variate Dirichlet type II distribution with parameters $\left(b_{1}, \ldots, b_{n} ; b_{n+1}\right)$, denoted by $\left(Y_{1}, \ldots, Y_{n}\right) \sim \mathbb{C} D_{m}^{I I}\left(b_{1}, \ldots, b_{n} ; b_{n+1}\right)$, if their joint p.d.f. is given by

$$
\frac{\tilde{\Gamma}_{m}\left(\sum_{i=1}^{n+1} b_{i}\right)}{\prod_{i=1}^{n+1} \tilde{\Gamma}_{m}\left(b_{i}\right)} \prod_{i=1}^{n} \operatorname{det}\left(Y_{i}\right)^{b_{i}-m} \operatorname{det}\left(I_{m}+\sum_{i=1}^{n} Y_{i}\right)^{-\sum_{i=1}^{n+1} b_{i}}
$$

where $b_{i}>m-1, i=1,2, \ldots, n+1$.

The type II density (6) can be obtained from the type I density (5) by transforming $Y_{i}=\left(I_{m}-\sum_{i=1}^{n} X_{i}\right)^{-\frac{1}{2}} X_{i}\left(I_{m}-\sum_{i=1}^{n} X_{i}\right)^{-\frac{1}{2}}, i=1, \ldots, n$ with the Jacobian $J\left(X_{1}, \ldots, X_{n} \rightarrow Y_{1}, \ldots, Y_{n}\right)=\operatorname{det}\left(I_{m}+\sum_{i=1}^{n} Y_{i}\right)^{-(n+1) m}$. It may also be noted here that $\sum_{i=1}^{n} X_{i} \sim \mathbb{C} B_{m}^{I}\left(\sum_{i=1}^{n} a_{i}, a_{n+1}\right)$ and $\sum_{i=1}^{n} Y_{i} \sim \mathbb{C} B_{m}^{I I}\left(\sum_{i=1}^{n} b_{i}, b_{n+1}\right)$.

In the next two theorems we derive the complex matrix variate Dirichlet distributions using independent complex Wishart matrices.

Theorem 1.8. Let $A_{i} \sim \mathbb{C} W_{m}\left(\nu_{i}, \Sigma\right), i=1, \ldots, n$, and $B \sim \mathbb{C} W_{m}(\mu, \Sigma)$ be independently distributed. Define

$$
X_{i}=A^{-\frac{1}{2}} A_{i}\left(A^{-\frac{1}{2}}\right)^{H}, \quad i=1, \ldots, n,
$$

where $A=\sum_{i=1}^{n} A_{i}+B$ and $A^{\frac{1}{2}}\left(A^{\frac{1}{2}}\right)^{H}$ is any reasonable factorization of $A$. Then $\left(X_{1}, \ldots, X_{n}\right) \sim \mathbb{C} D_{m}^{I}\left(\nu_{1}, \ldots, \nu_{n} ; \mu\right)$.

Proof. The joint density of $A_{1}, \ldots, A_{n}$ and $B$ is given by

$$
\begin{aligned}
\prod_{i=1}^{n}\left[\left\{\tilde{\Gamma}_{m}\left(\nu_{i}\right) \operatorname{det}(\Sigma)^{\nu_{i}}\right\}^{-1} \operatorname{etr}(\right. & \left.\left.-\Sigma^{-1} A_{i}\right) \operatorname{det}\left(A_{i}\right)^{\nu_{i}-m}\right] \\
& \times\left\{\tilde{\Gamma}_{m}(\mu) \operatorname{det}(\Sigma)^{\mu}\right\}^{-1} \operatorname{etr}\left(-\Sigma^{-1} B\right) \operatorname{det}(B)^{\mu-m} .
\end{aligned}
$$

Making the transformation $\sum_{i=1}^{n} A_{i}+B=A, A_{i}=A^{\frac{1}{2}} X_{i}\left(A^{\frac{1}{2}}\right)^{H}, i=1, \ldots, n$ with the Jacobian $J\left(A_{1}, \ldots, A_{n}, B \rightarrow X_{1}, \ldots, X_{n}, A\right)=\operatorname{det}(A)^{m n}$ in $(7)$, the joint density of $X_{1}, \ldots, X_{n}$ and $A$ is obtained as

$$
\begin{aligned}
\frac{\operatorname{det}(\Sigma)^{-(\mu+\nu)}}{\tilde{\Gamma}_{m}(\mu) \prod_{i=1}^{n} \tilde{\Gamma}_{m}\left(\nu_{i}\right)} \prod_{i=1}^{n} \operatorname{det}\left(X_{i}\right)^{\nu_{i}-m} \operatorname{det}\left(I_{m}-\right. & \left.\sum_{i=1}^{n} X_{i}\right)^{\mu-m} \\
& \times \operatorname{det}(A)^{\mu+\nu-m} \operatorname{etr}\left(-\Sigma^{-1} A\right),
\end{aligned}
$$

where $\nu=\sum_{i=1}^{n} \nu_{i}$. From (8), it is easily seen that $\left(X_{1}, \ldots, X_{n}\right)$ and $A$ are independent and the density of $\left(X_{1}, \ldots, X_{n}\right)$ is given by

$$
\frac{\tilde{\Gamma}_{m}(\mu+\nu)}{\tilde{\Gamma}_{m}(\mu) \prod_{i=1}^{n} \tilde{\Gamma}_{m}\left(\nu_{i}\right)} \prod_{i=1}^{n} \operatorname{det}\left(X_{i}\right)^{\nu_{i}-m} \operatorname{det}\left(I_{m}-\sum_{i=1}^{n} X_{i}\right)^{\mu-m}
$$

which is the desired result. 
For $n=1$, the above theorem gives the complex matrix variate beta type I distribution.

Theorem 1.9. Let $A_{i} \sim \mathbb{C} W_{m}\left(\nu_{i}, I_{m}\right), i=1, \ldots, n$, and $B \sim \mathbb{C} W_{m}\left(\mu, I_{m}\right)$ be independently distributed. Define

$$
Y_{i}=B^{-\frac{1}{2}} A_{i} B^{-\frac{1}{2}}, \quad i=1, \ldots, n,
$$

where $B^{\frac{1}{2}} B^{\frac{1}{2}}=B$. Then, $\left(Y_{1}, \ldots, Y_{n}\right) \sim \mathbb{C} D_{m}^{I I}\left(\nu_{1}, \ldots, \nu_{n} ; \mu\right)$.

Proof. The joint density of $A_{1}, \ldots, A_{n}$ and $B$ is given by

$$
\prod_{i=1}^{n}\left[\left\{\tilde{\Gamma}_{m}\left(\nu_{i}\right)\right\}^{-1} \operatorname{etr}\left(-A_{i}\right) \operatorname{det}\left(A_{i}\right)^{\nu_{i}-m}\right]\left\{\tilde{\Gamma}_{m}(\mu)\right\}^{-1} \operatorname{etr}(-B) \operatorname{det}(B)^{\mu-m} .
$$

Substituting $A_{i}=B^{\frac{1}{2}} Y_{i} B^{\frac{1}{2}}, i=1, \ldots, n$, with the Jacobian $J\left(A_{1}, \ldots, A_{n}, B \rightarrow\right.$ $\left.Y_{1}, \ldots, Y_{n}, B\right)=\operatorname{det}(B)^{m n}$ in $(9)$, the joint density of $Y_{1}, \ldots, Y_{n}$ and $B$ is derived as

$$
\left[\tilde{\Gamma}_{m}(\mu) \prod_{i=1}^{n} \tilde{\Gamma}_{m}\left(\nu_{i}\right)\right]^{-1} \prod_{i=1}^{n} \operatorname{det}\left(Y_{i}\right)^{\nu_{i}-m} \operatorname{etr}\left[-\left(I_{m}+\sum_{i=1}^{n} Y_{i}\right) B\right] \operatorname{det}(B)^{\mu+\nu-m}
$$

where $\nu=\sum_{i=1}^{n} \nu_{i}$. Integrating out $B$ using

$$
\begin{aligned}
\int_{B=B^{H}>0} \operatorname{etr}\left[-\left(I_{m}+\sum_{i=1}^{n} Y_{i}\right) B\right] \operatorname{det}(B)^{\mu+\nu-m} d B & \\
& =\tilde{\Gamma}_{m}(\mu+\nu) \operatorname{det}\left(I_{m}+\sum_{i=1}^{n} Y_{i}\right)^{-(\mu+\nu)}
\end{aligned}
$$

we get the desired result.

For $n=1$, the above theorem gives the complex matrix variate beta type II distribution.

\section{Factorizations}

In this section we derive factorizations of the complex matrix variate Dirichlet type I and type II densities.

Theorem 2.1. Let $\left(X_{1}, \ldots, X_{n}\right) \sim \mathbb{C} D_{m}^{I}\left(a_{1}, \ldots, a_{n} ; a_{n+1}\right)$ and define

$$
\begin{aligned}
U_{1} & =X_{1}, \\
U_{2} & =\left(I_{m}-X_{1}\right)^{-\frac{1}{2}} X_{2}\left(I_{m}-X_{1}\right)^{-\frac{1}{2}}, \\
& \vdots \\
U_{n} & =\left(I_{m}-X_{1}-\cdots-X_{n-1}\right)^{-\frac{1}{2}} X_{n}\left(I_{m}-X_{1}-\cdots-X_{n-1}\right)^{-\frac{1}{2}} .
\end{aligned}
$$


Then, the complex random matrices $U_{1}, \ldots, U_{n}$ are independently distributed, $U_{i} \sim \mathbb{C} B_{m}^{I}\left(a_{i}, \sum_{r=i+1}^{n+1} a_{r}\right), i=1, \ldots, n$.

Proof. The density of $\left(X_{1}, \ldots, X_{n}\right)$ is given by (5). From the above transformation it is easy to see that

$$
\begin{aligned}
\operatorname{det}\left(I_{m}-X_{1}\right) & =\operatorname{det}\left(I_{m}-U_{1}\right) \\
\operatorname{det}\left(I_{m}-X_{1}-X_{2}\right) & =\operatorname{det}\left(I_{m}-X_{1}-\left(I_{m}-X_{1}\right)^{\frac{1}{2}} U_{2}\left(I_{m}-X_{1}\right)^{\frac{1}{2}}\right), \\
& =\operatorname{det}\left(I_{m}-U_{1}\right) \operatorname{det}\left(I_{m}-U_{2}\right), \\
& \vdots \\
\operatorname{det}\left(I_{m}-X_{1}-\cdots-X_{n}\right) & =\operatorname{det}\left(I_{m}-U_{1}\right) \cdots \operatorname{det}\left(I_{m}-U_{n}\right), \\
\operatorname{det}\left(X_{1}\right) & =\operatorname{det}\left(U_{1}\right), \\
\operatorname{det}\left(X_{2}\right) & =\operatorname{det}\left(U_{2}\right) \operatorname{det}\left(I_{m}-U_{1}\right), \\
& \vdots \\
\operatorname{det}\left(X_{n}\right) & =\operatorname{det}\left(U_{n}\right) \operatorname{det}\left(I_{m}-U_{1}\right) \cdots \operatorname{det}\left(I_{m}-U_{n-1}\right),
\end{aligned}
$$

and

$$
\begin{aligned}
J\left(X_{1}, \ldots, X_{n} \rightarrow U_{1}, \ldots, U_{n}\right) & =\prod_{i=1}^{n-1} \operatorname{det}\left(I_{m}-\sum_{r=1}^{i} X_{r}\right)^{m} \\
& =\prod_{i=1}^{n-1} \operatorname{det}\left(I_{m}-U_{i}\right)^{m(n-i)}
\end{aligned}
$$

Substituting appropriately in the density of $\left(X_{1}, \ldots, X_{n}\right)$, one obtains the joint density of $U_{1}, \ldots, U_{n}$ as

$$
\begin{aligned}
& \frac{\tilde{\Gamma}_{m}\left(\sum_{i=1}^{n+1} a_{i}\right)}{\prod_{i=1}^{n+1} \tilde{\Gamma}_{m}\left(a_{i}\right)} \operatorname{det}\left(U_{1}\right)^{a_{1}-m} \operatorname{det}\left(I_{m}-U_{1}\right)^{a_{2}+\cdots+a_{n+1}-m} \\
& \times \operatorname{det}\left(U_{2}\right)^{a_{2}-m} \operatorname{det}\left(I_{m}-U_{2}\right)^{a_{3}+\cdots+a_{n+1}-m} \times \cdots \times \\
& \times \operatorname{det}\left(U_{n}\right)^{a_{n}-m} \operatorname{det}\left(I_{m}-U_{n}\right)^{a_{n+1}-m},
\end{aligned}
$$

where $0<U_{i}=U_{i}^{H}<I_{m}, i=1, \ldots, n$. Now, observing that

$$
\frac{\prod_{i=1}^{n+1} \tilde{\Gamma}_{m}\left(a_{i}\right)}{\tilde{\Gamma}_{m}\left(\sum_{i=1}^{n+1} a_{i}\right)}=\prod_{i=1}^{n} \tilde{B}_{m}\left(a_{i}, \sum_{r=i+1}^{n+1} a_{r}\right)
$$

we obtain the desired result. 
Theorem 2.2. Let $\left(X_{1}, \ldots, X_{n}\right) \sim \mathbb{C} D_{m}^{I}\left(a_{1}, \ldots, a_{n} ; a_{n+1}\right)$ and define

$$
\begin{aligned}
U_{n} & =X_{n}, \\
U_{n-1} & =\left(I_{m}-X_{n}\right)^{-\frac{1}{2}} X_{n-1}\left(I_{m}-X_{n}\right)^{-\frac{1}{2}}, \\
& \vdots \\
U_{1} & =\left(I_{m}-X_{n}-\cdots-X_{2}\right)^{-\frac{1}{2}} X_{1}\left(I_{m}-X_{n}-\cdots-X_{2}\right)^{-\frac{1}{2}} .
\end{aligned}
$$

Then $U_{1}, \ldots, U_{n}$ are independently distributed, $U_{i} \sim \mathbb{C} B_{m}^{I}\left(a_{i}, \sum_{r=1}^{i-1} a_{r}+a_{n+1}\right)$, $i=1, \ldots, n$.

Proof. Similar to the proof of Theorem 2.1.

Theorem 2.3. Let $\left(Y_{1}, \ldots, Y_{n}\right) \sim \mathbb{C} D_{m}^{I I}\left(b_{1}, \ldots, b_{n} ; b_{n+1}\right)$ and define

$$
\begin{aligned}
V_{n} & =Y_{n}, \\
V_{n-1} & =\left(I_{m}+Y_{n}\right)^{-\frac{1}{2}} Y_{n-1}\left(I_{m}+Y_{n}\right)^{-\frac{1}{2}}, \\
& \vdots \\
V_{1} & =\left(I_{m}+Y_{n}+\cdots+Y_{2}\right)^{-\frac{1}{2}} Y_{1}\left(I_{m}+Y_{n}+\cdots+Y_{2}\right)^{-\frac{1}{2}} .
\end{aligned}
$$

Then $V_{1}, \ldots, V_{n}$ are independently distributed, $V_{i} \sim \mathbb{C} B_{m}^{I I}\left(b_{i}, \sum_{r=i+1}^{n+1} b_{r}\right), i=1, \ldots, n$. Proof. Observe that

$$
\begin{aligned}
\operatorname{det}\left(I_{m}+Y_{n}\right) & =\operatorname{det}\left(I_{m}+V_{n}\right), \\
\operatorname{det}\left(I_{m}+Y_{n}+Y_{n-1}\right) & =\operatorname{det}\left(I_{m}+V_{n}\right) \operatorname{det}\left(I_{m}+V_{n-1}\right), \\
& \vdots \\
\operatorname{det}\left(I_{m}+Y_{n}+\cdots+Y_{1}\right) & =\operatorname{det}\left(I_{m}+V_{n}\right) \ldots \operatorname{det}\left(I_{m}+V_{1}\right),
\end{aligned}
$$

and

$$
\begin{aligned}
\operatorname{det}\left(Y_{n}\right) & =\operatorname{det}\left(V_{n}\right), \\
\operatorname{det}\left(Y_{n-1}\right) & =\operatorname{det}\left(V_{n-1}\right) \operatorname{det}\left(I_{m}+V_{n}\right), \\
& \vdots \\
\operatorname{det}\left(Y_{1}\right) & =\operatorname{det}\left(V_{1}\right) \operatorname{det}\left(I_{m}+V_{n}\right) \cdots \operatorname{det}\left(I_{m}+V_{2}\right) .
\end{aligned}
$$

Substituting these together with the Jacobian of transformation

$$
J\left(Y_{1}, \ldots, Y_{n} \rightarrow V_{1}, \ldots, V_{n}\right)=\prod_{r=2}^{n} \operatorname{det}\left(I_{m}+V_{r}\right)^{m(r-1)}
$$


in the density of $\left(Y_{1}, \ldots, Y_{n}\right)$ and simplifying, one obtains

$$
\frac{\tilde{\Gamma}_{m}\left(\sum_{i=1}^{n+1} b_{i}\right)}{\prod_{i=1}^{n+1} \tilde{\Gamma}_{m}\left(b_{i}\right)} \prod_{i=1}^{n}\left[\operatorname{det}\left(V_{i}\right)^{b_{i}-m} \operatorname{det}\left(I_{m}+V_{i}\right)^{-\sum_{r=i}^{n+1} b_{r}}\right],
$$

where $V_{i}=V_{i}^{H}>0, i=1, \ldots, n$. Now, using (12) the desired result follows.

Theorem 2.4. Let $\left(Y_{1}, \ldots, Y_{n}\right) \sim \mathbb{C} D_{m}^{I I}\left(b_{1}, \ldots, b_{n} ; b_{n+1}\right)$ and define

$$
\begin{aligned}
V_{1} & =Y_{1}, \\
V_{2} & =\left(I_{m}+Y_{1}\right)^{-\frac{1}{2}} Y_{2}\left(I_{m}+Y_{1}\right)^{-\frac{1}{2}}, \\
& \vdots \\
V_{n} & =\left(I_{m}+Y_{1}+\cdots+Y_{n-1}\right)^{-\frac{1}{2}} Y_{n}\left(I_{m}+Y_{1}+\cdots+Y_{n-1}\right)^{-\frac{1}{2}} .
\end{aligned}
$$

Then $V_{1}, \ldots, V_{n}$ are independently distributed, $V_{i} \sim \mathbb{C} B_{m}^{I I}\left(b_{i}, \sum_{r=1}^{i-1} b_{r}+b_{n+1}\right)$, $i=1, \ldots, n$.

Proof. Similar to the proof of Theorem 2.3.

The above results have been derived using matrix transformations. Tan [21] derived Theorem 2.2 and Theorem 2.4 using certain results on marginal and conditional distributions. Likewise, using suitable inverse transformations, one can derive the complex matrix variate Dirichlet distributions from the independent complex beta matrices as given in the following theorems.

Theorem 2.5. Let $U_{1}, \ldots, U_{n}$ be independent $m \times m$ complex random matrices, $U_{i} \sim \mathbb{C} B_{m}^{I}\left(\alpha_{i}, \beta_{i}\right), i=1, \ldots, n$. Define

$$
\begin{aligned}
X_{1} & =U_{1}, \\
X_{2} & =\left(I_{m}-U_{1}\right)^{\frac{1}{2}} U_{2}\left(I_{m}-U_{1}\right)^{\frac{1}{2}}, \\
& \vdots \\
X_{n} & =\left(I_{m}-U_{1}\right)^{\frac{1}{2}} \cdots\left(I_{m}-U_{n-1}\right)^{\frac{1}{2}} U_{n}\left(I_{m}-U_{n-1}\right)^{\frac{1}{2}} \cdots\left(I_{m}-U_{1}\right)^{\frac{1}{2}} .
\end{aligned}
$$

Then, $\left(X_{1}, \ldots, X_{n}\right) \sim \mathbb{C} D_{m}^{I}\left(\alpha_{1}, \ldots, \alpha_{n} ; \beta_{n}\right)$ iff $\beta_{i}=\alpha_{i+1}+\beta_{i+1}, i=1, \ldots, n-1$.

Theorem 2.6. Let $U_{1}, \ldots, U_{n}$ be independent $m \times m$ random matrices, and $U_{i} \sim$ $\mathbb{C} B_{m}^{I}\left(\alpha_{i}, \beta_{i}\right), i=1, \ldots, n$. Define

$$
\begin{aligned}
X_{n} & =U_{n}, \\
X_{n-1} & =\left(I_{m}-U_{n}\right)^{\frac{1}{2}} U_{n-1}\left(I_{m}-U_{n}\right)^{\frac{1}{2}}, \\
& \vdots \\
X_{1} & =\left(I_{m}-U_{n}\right)^{\frac{1}{2}} \cdots\left(I_{m}-U_{2}\right)^{\frac{1}{2}} U_{1}\left(I_{m}-U_{2}\right)^{\frac{1}{2}} \cdots\left(I_{m}-U_{n}\right)^{\frac{1}{2}} .
\end{aligned}
$$


Then, $\left(X_{1}, \ldots, X_{n}\right) \sim \mathbb{C} D_{m}^{I}\left(\alpha_{1}, \ldots, \alpha_{n} ; \beta_{1}\right)$ iff $\beta_{i+1}=\alpha_{i}+\beta_{i}, i=1, \ldots, n-1$.

Theorem 2.7. Let $V_{1}, \ldots, V_{n}$ be independent $m \times m$ complex random matrices, $V_{i} \sim \mathbb{C} B_{m}^{I I}\left(\alpha_{i}, \beta_{i}\right), i=1, \ldots, n$. Define

$$
\begin{aligned}
Y_{n} & =V_{n}, \\
Y_{n-1} & =\left(I_{m}+V_{n}\right)^{\frac{1}{2}} V_{n-1}\left(I_{m}+V_{n}\right)^{\frac{1}{2}}, \\
& \vdots \\
Y_{1} & =\left(I_{m}+V_{n}\right)^{\frac{1}{2}} \cdots\left(I_{m}+V_{2}\right)^{\frac{1}{2}} V_{1}\left(I_{m}+V_{2}\right)^{\frac{1}{2}} \cdots\left(I_{m}+V_{n}\right)^{\frac{1}{2}} .
\end{aligned}
$$

Then, $\left(Y_{1}, \ldots, Y_{n}\right) \sim \mathbb{C} D_{m}^{I I}\left(\alpha_{1}, \ldots, \alpha_{n} ; \beta_{n}\right)$ iff $\beta_{i}=\alpha_{i+1}+\beta_{i+1}, i=1, \ldots, n-1$.

Theorem 2.8. Let $V_{1}, \ldots, V_{n}$ be independent $m \times m$ complex random matrices, and $V_{i} \sim \mathbb{C} B_{m}^{I I}\left(\alpha_{i}, \beta_{i}\right), i=1, \ldots, n$. Define

$$
\begin{aligned}
Y_{1} & =V_{1}, \\
Y_{2} & =\left(I_{m}+V_{1}\right)^{\frac{1}{2}} V_{2}\left(I_{m}+V_{1}\right)^{\frac{1}{2}}, \\
& \vdots \\
Y_{n} & =\left(I_{m}+V_{1}\right)^{\frac{1}{2}} \cdots\left(I_{m}+V_{n-1}\right)^{\frac{1}{2}} V_{n}\left(I_{m}+V_{n-1}\right)^{\frac{1}{2}} \cdots\left(I_{m}+V_{1}\right)^{\frac{1}{2}} .
\end{aligned}
$$

Then $\left(Y_{1}, \ldots, Y_{n}\right) \sim \mathbb{C} D_{m}^{I I}\left(\alpha_{1}, \ldots, \alpha_{n} ; \beta_{1}\right)$ iff $\beta_{i+1}=\alpha_{i}+\beta_{i}, i=1, \ldots, n-1$.

From the transformation given in Theorem 2.5, one can see that

$$
I_{m}-\sum_{i=1}^{n} X_{i}=\left(I_{m}-U_{1}\right)^{\frac{1}{2}} \cdots\left(I_{m}-U_{n-1}\right)^{\frac{1}{2}}\left(I_{m}-U_{n}\right)\left(I_{m}-U_{n-1}\right)^{\frac{1}{2}} \cdots\left(I_{m}-U_{1}\right)^{\frac{1}{2}}
$$

where $I_{m}-U_{1}, \ldots, I_{m}-U_{n}$ are independent, $I_{m}-U_{i} \sim \mathbb{C} B_{m}^{I}\left(\beta_{i}, \alpha_{i}\right), i=1, \ldots, n$ and $I_{m}-\sum_{i=1}^{n} X_{i} \sim \mathbb{C} B_{m}^{I}\left(\beta_{n}, \sum_{i=1}^{n} \alpha_{i}\right)$ iff $\beta_{i}=\alpha_{i+1}+\beta_{i+1}, i=1, \ldots, n-1$. Similarly, from Theorem 2.6, one obtains

$$
I_{m}-\sum_{i=1}^{n} X_{i}=\left(I_{m}-U_{n}\right)^{\frac{1}{2}} \cdots\left(I_{m}-U_{2}\right)^{\frac{1}{2}}\left(I_{m}-U_{1}\right)\left(I_{m}-U_{2}\right)^{\frac{1}{2}} \cdots\left(I_{m}-U_{n}\right)^{\frac{1}{2}}
$$

where $I_{m}-U_{1}, \ldots, I_{m}-U_{n}$ are independent, $I_{m}-U_{i} \sim \mathbb{C} B_{m}^{I}\left(\beta_{i}, \alpha_{i}\right), i=1, \ldots, n$ and $I_{m}-\sum_{i=1}^{n} X_{i} \sim \mathbb{C} B_{m}^{I}\left(\beta_{1}, \sum_{i=1}^{n} \alpha_{i}\right)$ iff $\beta_{i+1}=\alpha_{i}+\beta_{i}, i=1, \ldots, n-1$. Thus, we obtain the following result generalizing a result given in Gupta and Nagar [9].

Theorem 2.9. Let $W_{1}, \ldots, W_{n}$ be independent $m \times m$ complex random matrices, $W_{i} \sim \mathbb{C} B_{m}^{I}\left(c_{i}, d_{i}\right), i=1, \ldots, n$. Then

$$
W_{1}^{\frac{1}{2}} \cdots W_{n-1}^{\frac{1}{2}} W_{n} W_{n-1}^{\frac{1}{2}} \cdots W_{1}^{\frac{1}{2}} \sim \mathbb{C} B_{m}^{I}\left(c_{n}, \sum_{i=1}^{n} d_{i}\right)
$$


iff $c_{i}=d_{i+1}+c_{i+1}, i=1 \ldots, n-1$ and

$$
W_{n}^{\frac{1}{2}} \cdots W_{2}^{\frac{1}{2}} W_{1} W_{2}^{\frac{1}{2}} \cdots W_{n}^{\frac{1}{2}} \sim \mathbb{C} B_{m}^{I}\left(c_{1}, \sum_{i=1}^{n} d_{i}\right)
$$

iff $c_{i+1}=d_{i}+c_{i}, i=1 \ldots, n-1$.

Corollary 2.10. Let $W_{1}$ and $W_{2}$ be independent, $W_{1} \sim \mathbb{C} B_{m}^{I}\left(c_{1}, d_{1}\right)$ and $W_{2} \sim$ $\mathbb{C} B_{m}^{I}\left(c_{1}+d_{1}, d_{2}\right)$. Then $W_{2}^{\frac{1}{2}} W_{1} W_{2}^{\frac{1}{2}} \sim \mathbb{C} B_{m}^{I}\left(c_{1}, d_{1}+d_{2}\right)$.

The above result, in the real case, was obtained by Javier and Gupta [12]. This result can also be derived as a corollary of the following theorem.

Theorem 2.11. Let $W_{1}$ and $W_{2}$ be independent, $W_{1} \sim \mathbb{C} B_{m}^{I}\left(c_{1}, d_{1}\right)$ and $W_{2} \sim$ $\mathbb{C} B_{m}^{I}\left(c_{2}, d_{2}\right)$. Then, the p.d.f. of $U=W_{2}^{\frac{1}{2}} W_{1} W_{2}^{\frac{1}{2}}$ is given by

$$
\begin{aligned}
& \frac{\tilde{\Gamma}_{m}\left(c_{1}+d_{1}\right) \tilde{\Gamma}_{m}\left(c_{2}+d_{2}\right)}{\tilde{\Gamma}_{m}\left(c_{1}\right) \tilde{\Gamma}_{m}\left(c_{2}\right) \tilde{\Gamma}_{m}\left(d_{1}+d_{2}\right)} \operatorname{det}(U)^{c_{1}-m} \operatorname{det}\left(I_{m}-U\right)^{d_{1}+d_{2}-m} \\
& \quad \times{ }_{2} \tilde{F}_{1}\left(c_{1}+d_{1}-c_{2}, d_{2} ; d_{1}+d_{2} ; I_{m}-U\right), \quad 0<U=U^{H}<I_{m},
\end{aligned}
$$

where ${ }_{2} \tilde{F}_{1}$ is the Gauss hypergeometric function of the Hermitian matrix argument.

Proof. The joint density of $W_{1}$ and $W_{2}$ is

$$
\begin{aligned}
& \left\{\tilde{B}_{m}\left(c_{1}, d_{1}\right) \tilde{B}_{m}\left(c_{2}, d_{2}\right)\right\}^{-1} \operatorname{det}\left(W_{1}\right)^{c_{1}-m} \operatorname{det}\left(I_{m}-W_{1}\right)^{d_{1}-m} \operatorname{det}\left(W_{2}\right)^{c_{2}-m} \\
& \quad \times \operatorname{det}\left(I_{m}-W_{2}\right)^{d_{2}-m}, \quad 0<W_{1}=W_{1}^{H}<I_{m}, \quad 0<W_{2}=W_{2}^{H}<I_{m} .
\end{aligned}
$$

Making the transformation $U=W_{2}^{\frac{1}{2}} W_{1}\left(W_{2}^{\frac{1}{2}}\right)^{H}$ with the Jacobian $J\left(W_{1}, W_{2} \rightarrow\right.$ $\left.U, W_{2}\right)=\operatorname{det}\left(W_{2}\right)^{-m}$ in (13) we get the joint density of $U$ and $W_{2}$ as

$$
\begin{aligned}
& \left\{\tilde{B}_{m}\left(c_{1}, d_{1}\right) \tilde{B}_{m}\left(c_{2}, d_{2}\right)\right\}^{-1} \operatorname{det}(U)^{c_{1}-m} \operatorname{det}\left(I_{m}-W_{2}^{-\frac{1}{2}} U\left(W_{2}^{-\frac{1}{2}}\right)^{H}\right)^{d_{1}-m} \\
& \quad \times \operatorname{det}\left(W_{2}\right)^{c_{2}-c_{1}-m} \operatorname{det}\left(I_{m}-W_{2}\right)^{d_{2}-m}, \quad 0<U=U^{H}<W_{2}=W_{2}^{H}<I_{m} .
\end{aligned}
$$

Now to obtain the marginal density of $U$, we need to evaluate

$$
\begin{aligned}
\int_{U<W_{2}=W_{2}^{H}<I_{m}} \operatorname{det}\left(I_{m}-W_{2}^{-\frac{1}{2}}\right. & \left.U\left(W_{2}^{-\frac{1}{2}}\right)^{H}\right)^{d_{1}-m} \\
& \times \operatorname{det}\left(W_{2}\right)^{c_{2}-c_{1}-m} \operatorname{det}\left(I_{m}-W_{2}\right)^{d_{2}-m} d W_{2} .
\end{aligned}
$$


Substituting in (15), $W=\left(I_{m}-U\right)^{-\frac{1}{2}}\left(W_{2}-U\right)\left(\left(I_{m}-U\right)^{-\frac{1}{2}}\right)^{H}$ with the Jacobian $J\left(W_{2} \rightarrow W\right)=\operatorname{det}\left(I_{m}-U\right)^{m}$, we get

$$
\begin{aligned}
& \operatorname{det}\left(I_{m}-U\right)^{d_{1}+d_{2}-m} \int_{0<W=W^{H}<I_{m}} \operatorname{det}(W)^{d_{1}-m} \operatorname{det}\left(I_{m}-W\right)^{d_{2}-m} d W \\
& \times \operatorname{det}\left(I_{m}-\left(I_{m}-U\right)(I-W)\right)^{-\left(c_{1}+d_{1}-c_{2}\right)} d W \\
& =\operatorname{det}\left(I_{m}-U\right)^{d_{1}+d_{2}-m} \frac{\tilde{\Gamma}_{m}\left(d_{1}\right) \tilde{\Gamma}_{m}\left(d_{2}\right)}{\tilde{\Gamma}_{m}\left(d_{1}+d_{2}\right)}{ }_{2} \tilde{F}_{1}\left(c_{1}+d_{1}-c_{2}, d_{2} ; d_{1}+d_{2} ; I_{m}-U\right)
\end{aligned}
$$

where the integration has been carried out using the integral representation of the Gauss hypergeometric function of the Hermitian matrix argument (James [11], Chikuse [4]). Integration of $W_{2}$ in (14), using (15) and (16), completes the proof of the theorem.

Now, by substituting $c_{1}+d_{1}=c_{2}$ in Theorem 2.11 we get Corollary 2.10. In the following theorem, further generalization of Corollary 2.10 is obtained by replacing complex beta type I matrix by a set of complex Dirichlet type I matrices.

Theorem 2.12. Let $A$ and $\left(X_{1}, \ldots, X_{n}\right)$ be independent, $A \sim \mathbb{C} B_{m}^{I}\left(\sum_{i=1}^{n+1} a_{i}, c\right)$ and $\left(X_{1}, \ldots, X_{n}\right) \sim \mathbb{C} D_{m}^{I}\left(a_{1}, \ldots, a_{n} ; a_{n+1}\right)$. Then,

$$
\left(A^{\frac{1}{2}} X_{1} A^{\frac{1}{2}}, \ldots, A^{\frac{1}{2}} X_{n} A^{\frac{1}{2}}\right) \sim \mathbb{C} D_{m}^{I}\left(a_{1}, \ldots, a_{n} ; a_{n+1}+c\right) .
$$

Proof. The joint density of $A$ and $\left(X_{1}, \ldots, X_{n}\right)$ is given by

$$
\begin{aligned}
\frac{\tilde{\Gamma}_{m}\left(\sum_{i=1}^{n+1} a_{i}+c\right)}{\prod_{i=1}^{n+1} \tilde{\Gamma}_{m}\left(a_{i}\right) \tilde{\Gamma}_{m}(c)} \prod_{i=1}^{n} \operatorname{det}\left(X_{i}\right)^{a_{i}-m} \operatorname{det}\left(I_{m}\right. & \left.-\sum_{i=1}^{n} X_{i}\right)^{a_{n+1}-m} \\
& \times \operatorname{det}(A)^{\sum_{i=1}^{n+1} a_{i}-m} \operatorname{det}\left(I_{m}-A\right)^{c-m} .
\end{aligned}
$$

Substituting $U_{i}=A^{\frac{1}{2}} X_{i} A^{\frac{1}{2}}, i=1, \ldots, n$ with the Jacobian $J\left(X_{1}, \ldots, X_{n}, A \rightarrow\right.$ $\left.U_{1}, \ldots, U_{n}, A\right)=\operatorname{det}(A)^{-m n}$ in the above expression, we obtain the joint density of $\left(U_{1}, \ldots, U_{n}\right)$ and $A$ as

$$
\frac{\tilde{\Gamma}_{m}\left(\sum_{i=1}^{n+1} a_{i}+c\right)}{\prod_{i=1}^{n+1} \tilde{\Gamma}_{m}\left(a_{i}\right) \tilde{\Gamma}_{m}(c)} \prod_{i=1}^{n} \operatorname{det}\left(U_{i}\right)^{a_{i}-m} \operatorname{det}\left(A-\sum_{i=1}^{n} U_{i}\right)^{a_{n+1}-m} \operatorname{det}\left(I_{m}-A\right)^{c-m}
$$

where $0<\sum_{i=1}^{n} U_{i}<A=A^{H}<I_{m}$. Now, transforming $Z=\left(I_{m}-\sum_{i=1}^{n} U_{i}\right)^{-\frac{1}{2}}(A-$ $\left.\sum_{i=1}^{n} U_{i}\right)\left(I_{m}-\sum_{i=1}^{n} U_{i}\right)^{-\frac{1}{2}}$ with the Jacobian $J(A \rightarrow Z)=\operatorname{det}\left(I_{m}-\sum_{i=1}^{n} U_{i}\right)^{m}$ the joint density of $\left(U_{1}, \ldots, U_{n}\right)$ and $Z$ is derived as

$$
\begin{aligned}
\frac{\tilde{\Gamma}_{m}\left(\sum_{i=1}^{n+1} a_{i}+c\right)}{\prod_{i=1}^{n} \tilde{\Gamma}_{m}\left(a_{i}\right) \tilde{\Gamma}_{m}\left(a_{n+1}+c\right)} \prod_{i=1}^{n} \operatorname{det}\left(U_{i}\right)^{a_{i}-m} \operatorname{det}\left(I_{m}-\sum_{i=1}^{n} U_{i}\right)^{a_{n+1}+c-m} \\
\times\left\{\tilde{B}\left(a_{n+1}, c\right)\right\}^{-1} \operatorname{det}(Z)^{a_{n+1}-m} \operatorname{det}\left(I_{m}-Z\right)^{c-m}
\end{aligned}
$$


where the matrices $U_{1}, \ldots, U_{n}, I_{m}-\sum_{i=1}^{n} U_{i}, Z$, and $I_{m}-Z$ are Hermitian positive definite.

Corollary 2.13. Let $B$ and $\left(X_{1}, \ldots, X_{n}\right)$ be independent, $B \sim \mathbb{C} B_{m}^{I I}\left(c, \sum_{i=1}^{n+1} a_{i}\right)$ and $\left(X_{1}, \ldots, X_{n}\right) \sim \mathbb{C} D_{m}^{I}\left(a_{1}, \ldots, a_{n} ; a_{n+1}\right)$. Then,

$$
\left((I+B)^{-\frac{1}{2}} X_{1}(I+B)^{-\frac{1}{2}}, \ldots,(I+B)^{-\frac{1}{2}} X_{n}(I+B)^{-\frac{1}{2}}\right) \sim \mathbb{C} D_{m}^{I}\left(a_{1}, \ldots, a_{n} ; a_{n+1}+c\right) .
$$

The following result is obtained by replacing complex beta type I matrix by a complex Wishart matrix in Theorem 2.12.

Theorem 2.14. Let $A$ and $\left(X_{1}, \ldots, X_{n}\right)$ be independent, $A \sim \mathbb{C} W_{m}\left(\sum_{i=1}^{n+1} a_{i}, \Sigma\right)$ and $\left(X_{1}, \ldots, X_{n}\right) \sim \mathbb{C} D_{m}^{I}\left(a_{1}, \ldots, a_{n} ; a_{n+1}\right)$. Define $W_{i}=A^{\frac{1}{2}} X_{i} A^{\frac{1}{2}}, i=1, \ldots, n-1$, and $W_{n}=A^{\frac{1}{2}}\left(I_{m}-\sum_{i=1}^{n} X_{i}\right) A^{\frac{1}{2}}$. Then, $W_{1}, \ldots, W_{n}$ are independent, $W_{i} \sim \mathbb{C} W_{m}\left(a_{i}, \Sigma\right)$, $i=1, \ldots, n-1$, and $W_{n} \sim \mathbb{C} W_{m}\left(a_{n+1}, \Sigma\right)$.

Proof. The joint density of $A$ and $\left(X_{1}, \ldots, X_{n}\right)$ is given by

$$
\begin{aligned}
{\left[\prod_{i=1}^{n+1} \tilde{\Gamma}_{m}\left(a_{i}\right) \operatorname{det}(\Sigma)^{\sum_{i=1}^{n+1} a_{i}}\right]^{-1} \prod_{i=1}^{n} \operatorname{det}\left(X_{i}\right)^{a_{i}-m} } & \operatorname{det}\left(I_{m}-\sum_{i=1}^{n} X_{i}\right)^{a_{n+1}-m} \\
& \times \operatorname{det}(A)^{\sum_{i=1}^{n+1} a_{i}-m} \operatorname{etr}\left(-\Sigma^{-1} A\right) .
\end{aligned}
$$

Substituting $W_{i}=A^{\frac{1}{2}} X_{i} A^{\frac{1}{2}}, i=1, \ldots, n-1$, and $W_{n}=A^{\frac{1}{2}}\left(I_{m}-\sum_{i=1}^{n} X_{i}\right) A^{\frac{1}{2}}$ with the Jacobian $J\left(X_{1}, \ldots, X_{n}, A \rightarrow W_{1}, \ldots, W_{n}, A\right)=\operatorname{det}(A)^{-m n}$ in the above expression, we obtain the joint density of $\left(W_{1}, \ldots, W_{n}\right)$ and $A$ as

$$
\left[\prod_{i=1}^{n+1} \tilde{\Gamma}_{m}\left(a_{i}\right) \operatorname{det}(\Sigma)^{\sum_{i=1}^{n+1} a_{i}}\right]^{-1} \prod_{i=1}^{n-1} \operatorname{det}\left(W_{i}\right)^{a_{i}-m} \operatorname{det}\left(W_{n}\right)^{a_{n+1}-m} \operatorname{etr}\left(-\Sigma^{-1} A\right)
$$

where $W_{1}, \ldots, W_{n}$ and $A$ are Hermitian positive definite with $\sum_{i=1}^{n} W_{i}<A$. The desired result now follows by substituting $Z=A-\sum_{i=1}^{n} W_{i}$.

Corollary 2.15. Let $X \sim \mathbb{C} B_{m}^{I}\left(a_{1}, a_{2}\right)$ and $A \sim \mathbb{C} W_{m}\left(a_{1}+a_{2}, \Sigma\right)$ be independent. Define $W_{1}=A^{\frac{1}{2}} X A^{\frac{1}{2}}$ and $W_{2}=A^{\frac{1}{2}}\left(I_{m}-X\right) A^{\frac{1}{2}}$. Then, $W_{1}$ and $W_{2}$ are independent, $W_{1} \sim \mathbb{C} W_{m}\left(a_{1}, \Sigma\right)$ and $W_{2} \sim \mathbb{C} W_{m}\left(a_{2}, \Sigma\right)$.

\section{References}

[1] B. V. Bronk, Ensemble for random matrices, J. Math. Phys. 6 (1965), no. 2, 228-237.

[2] D. R. Brillinger, Asymptotic properties of spectral estimates of second order, Biometrika 56 (1969), 375-390.

[3] M. Carmeli, Statistical theory and random matrices, Monographs and Textbooks in Pure and Applied Mathematics, vol. 74, Marcel Dekker Inc., New York, 1983. 
[4] Y. Chikuse, Partial differential equations for hypergeometric functions of complex argument matrices and their applications, Ann. Inst. Statist. Math. 28 (1976), no. 2, 187-199.

[5] W. J. Conradie and A. K. Gupta, Quadratic forms in complex normal variates: basic results, Statistica (Bologna) 47 (1987), no. 1, 73-84.

[6] N. R. Goodman, Statistical analysis based on a certain multivariate complex Gaussian distribution (an introduction), Ann. Math. Statist. 34 (1963), 152-177.

[7] _ The distribution of the determinant of a complex Wishart distributed matrix, Ann. Math. Statist. 34 (1963), 178-180.

[8] A. K. Gupta and D. K. Nagar, Distribution of the product of determinants of random matrices connected with the noncentral matrix variate Dirichlet distribution, South African Statist. J. 21 (1987), no. 2, 141-153.

[9] _ Matrix variate distributions, Chapman \& Hall/CRC Monographs and Surveys in Pure and Applied Mathematics, vol. 104, Chapman \& Hall/CRC, Boca Raton, FL, 2000.

[10] T. Hayakawa, On the distribution of the latent roots of a complex Wishart matrix (non-central case), Ann. Inst. Statist. Math. 24 (1972), 1-17.

[11] A. T. James, Distributions of matrix variates and latent roots derived from normal samples, Ann. Math. Statist. 35 (1964), 475-501.

[12] W. R. Javier and A. K. Gupta, On generalized matric variate beta distributions, Statistics 16 (1985), no. 4, 549-557.

[13] C. G. Khatri, Classical statistical analysis based on a certain multivariate complex Gaussian distribution, Ann. Math. Statist. 36 (1965), 98-114.

[14] mal vectors, Ann. Math. Statist 37 (1966), 468-479.

[15] P. R. Krishnaiah, Some recent developments on complex multivariate distributions, J. Multivariate Anal. 6 (1976), no. 1, 1-30.

[16] M. L. Mehta, Random matrices, Academic Press Inc., Boston, MA, 1991.

[17] D. K. Nagar and E. L. Arias, Complex matrix variate Cauchy distribution, Sci. Math. Jpn. 58 (2003), no. $1,67-80$.

[18] I. Olkin and H. Rubin, Multivariate beta distributions and independence properties of the Wishart distribution, Ann. Math. Statist 35 (1964), 261-269. Correction (1966), 37(1), 297.

[19] P. J. Smith and H. Gao, A determinant representation for the distribution of a generalised quadratic form in complex normal vectors, J. Multivariate Anal. 73 (2000), no. 1, 41-54.

[20] M. S. Srivastava, On the complex Wishart distribution, Ann. Math. Statist. 36 (1965), 313-315.

[21] W. Y. Tan, Some distribution theory associated with complex Gaussian distribution, Tamkang J. 7 (1968), 263-302.

[22] _ Note on the multivariate and the generalized multivariate beta distributions, J. Amer. Statist. Assoc. 64 (1969), 230-241.

[23] G. L. Turin, The characteristic function of Hermitian quadratic forms in complex normal variables, Biometrika 47 (1960), 199-201.

[24] S. S. Wilks, Mathematical statistics, A Wiley Publication in Mathematical Statistics, John Wiley \& Sons Inc., New York, 1962.

[25] R. A. Wooding, The multivariate distribution of complex normal variables, Biometrika 43 (1956), 212-215. 